\title{
LOS ESTILOS DE AMOR Y SU RELACIÓN CON EL BIENESTAR EN PERSONAS CON PAREJAS MONÓGAMAS Y NO-MONÓGAMAS
}

\author{
Alicia Tamarit Chuliá. \\ Dpto. Personalidad, Evaluación y Tratamientos Psicológicos. Universitat de València \\ alicia.tamarit@uv.es \\ Estefanía Mónaco Gerónimo. \\ Dpto. Personalidad, Evaluación y Tratamientos Psicológicos. Universitat de València. \\ estefania.monaco@uv.es \\ Alejandro Sánchez Ferrer. \\ Dpto. Personalidad, Evaluación y Tratamientos Psicológicos. Universitat de València. \\ aferrer@cop.es
}

\section{RESUMEN}

El estudio las relaciones sentimentales en la literatura científica ha centrado su atención en el bienestar debido a su importancia en la salud mental, y por ello ha sido de especial interés su relación con los estilos de amor y la diversidad en la forma de establecer vínculos afectivo-sexuales. El objetivo fue analizar la relación entre los estilos de amor $\mathrm{y}$ el bienestar, y las diferencias en función de la orientación afectiva (personas con parejas monógamas y no-monógamas) en estas variables. Participaron 168 personas con edades comprendidas entre los 16 y los 48 años $(M=26.24 ; D T=5.76)$. Se utilizó La Escala de Afectos Positivos y Negativos (SPANE), la Escala de Satisfacción con la Vida (SWLS) y la Escala de Actitudes hacia el Amor (LAS). Se realizaron análisis descriptivos, correlaciones bivariadas y análisis de regresión lineal jerárquica para analizar la relación entre las variables y pruebas $\mathrm{T}$ para estudiar las diferencias de medias. Los resultados indican que los estilos de amor están significativamente relacionados con el bienestar: Eros tiene una relación positiva, mientras que Ludus, Mania y Agape correlacionan de forma negativa. Un análisis de regresión jerárquica indicó que el género y la edad junto con los estilos de amor explican un $11.20 \%$ de la satisfacción con la vida, $11.30 \%$ de los afectos positivos y un $12.50 \%$ de los afectos negativos, siendo Eros, Mania y Agape los predictores más significativos. Los análisis de diferencias de medias mostraron que las personas con parejas monógamas presentan niveles más altos que las no-monógamas en Eros, Ludus y Pragma. Estos resultados enfatizan la importancia de estudiar el establecimiento de vínculos afectivo-sexuales desde una perspectiva diversa, teniendo en cuenta la variabilidad en los estilos afectivos y las diferencias en función de la orientación afectiva, ya que pueden jugar un importante papel en el bienestar subjetivo.

Palabras clave: estilos de amor, bienestar, diversidad afectiva, monogamia, no monogamia. 
TITLE. LOVE STYLES AND THEIR RELATIONSHIP TO WELL-BEING IN PEOPLE WITH MONOGAMOUS AND NON-MONOGAMOUS PARTNERS

\begin{abstract}
The study of romantic relationships in the scientific literature has focused on well-being due to its importance in mental health. For this reason, its relationship with love styles and diversity in the way of establishing affective-sexual bonds has been of particular interest. The aim was to analyze the relationship between love styles and well-being, and the differences according to affective orientation (people with monogamous and non-monogamous partners) in these variables. A total of 168 people between the ages of 16 and 48 years $(M=26.24 ; S D=5.76)$ participated. The Positive and Negative Affect Scale (SPANE), the Satisfaction with Life Scale (SWLS) and the Love Attitude Scale (LAS) were used. Descriptive analyses, bivariate correlations and hierarchical linear regression analyses were performed to analyze the relationship between the variables and t-tests to study mean differences. The results indicate that love styles are significantly related to well-being: Eros has a positive relationship, while Ludus, Mania and Agape correlate negatively. A hierarchical regression analysis indicated that gender and age together with love styles explain $11.20 \%$ of life satisfaction, $11.30 \%$ of positive affect and $12.50 \%$ of negative affect, with Eros, Mania and Agape being the most significant predictors. The differential analyses showed that people with monogamous partners had higher levels of Eros, Ludus and Pragma than non-monogamous partners. These results emphasize the importance of studying the establishment of affective-sexual bonds from a diverse perspective, considering the variability in affective styles and differences according to affective orientation, as they may play an important role in subjective well-being.
\end{abstract}

Key words: love styles, well-being, affective diversity, monogamy, non-monogamy. 


\section{Introducción}

El bienestar psicológico es uno de los constructos más estudiados en la literatura en psicología, y engloba todas aquellas emociones, sensaciones y experiencias relacionadas a la percepción subjetiva de la propia vida, y la valoración que se hace de ella a nivel global (Proctor et al., 2009). Se ha estudiado cómo la combinación de la satisfacción con la vida y los afectos positivos y negativos, así como el balance afectivo que es el resultado de la suma de estos últimos (Schoeps et al., 2019).

Debido a la complejidad de este concepto, se ha estudiado desde perspectivas muy distintas, y se ha relacionado con diversas variables a nivel psicológico et al., 2015). Concretamente, uno de los puntos de vista más estudiados ha sido en el contexto de las relaciones interpersonales, como resultado de la interacción social y teniendo en cuenta las emociones asociadas a estas relaciones (Ronen et al., 2016). Por ello, una de las variables con la que se ha relacionado el bienestar ha sido el amor, especialmente el amor romántico (Kansky, 2018).

Entre los estudios sobre amor romántico, una de las variables más estudiadas ha sido los estilos de amor (Jonason \& Kavanagh, 2010; Walsh, 2017). La clasificación de arquetipos amatorios de Lee (1977) ha sido referente en esta rama de la psicología, retomada por Hendrick y Dicke en 1998. Define seis estilos de amor, tres primarios (Eros, Ludus y Storge) y tres secundarios, que resultan de la combinación de los tres anteriores (Agape, Mania y Pragma) (Lee, 1977). Entre los primarios, Eros se define por mayores niveles de intimidad y pasión, predomina la atracción física. En segundo lugar, Storge se caracteriza por la importancia que adquieren la intimidad y el compromiso; la afinidad de gustos y valores se encuentra en un punto mayor en la escala de prioridades. En Ludus hay un nivel de compromiso más reducido, es un estilo de amor apasionado, basado en la atracción estética. En cuanto a los estilos secundarios, Agape (una combinación de Eros y Storge) es un amor desinteresado, altruista, basado en complacer a la pareja o las parejas; Mania (suma de Eros y Ludus) es un estilo más asociado a los celos y la posesividad, relacionado con la dependencia emocional y patrones de apego poco saludables; por último, Pragma (una combinación de Storge y Ludus) es un estilo de amor basado en expectativas y exigencias que giran en torno a un ideal de pareja, un estereotipo de amor romántico.

Si bien esta clasificación de estilos de amor ha sido ampliamente utilizada en psicología, su concepción teórica deja fuera muchas realidades que existen en las relaciones afectivo-sexuales (Hendrick et al., 1998). La perspectiva tradicional a la hora de diseñar modelos de amor romántico en la literatura suele ser, por un lado, centrada en la monogamia (lo que en literatura se denomina "mononormatividad"), ignorando otros estilos relacionales que caen en puntos distintos del abanico de diversidad afectiva (Sizemore \& Olmstead, 2017). Además, es generalmente alocentrista, lo que implica que se asume que las personas son por defecto alosexuales (es decir, que sienten atracción sexual por otras personas) e invisibiliza otras realidades dentro del espectro de la asexualidad (Gressgård, 2013). Por último, las visiones tradicionales en este ámbito suelen ser cisheterocentristas, dejando fuera la multiplicidad de realidades dentro del colectivo LGTBIQ+ (Thorne et al., 2019).

Existe un gran vacío en la literatura en cuanto al estudio de la diversidad afectiva y su relación con las concepciones tradicionales del amor romántico (Sizemore \& Olmstead, 2017). Tradicionalmente considerada como sinónimo de "poliamor", la orientación afectiva es un espectro que abarca diversos estilos relacionales, de modo 
que en los últimos años se ha utilizado el término paraguas de "no-monogamia" para abarcar toda esta diversidad de formas de relacionarse a nivel afectivo-sexual (De las Heras, 2019).

Una de las clasificaciones más populares consiste en un espectro que ordena estas orientaciones según su nivel de jerarquización de las estructuras relacionales, de mayor a menor, como sugieren Sheff y Tesene (2015). Así, en un extremo estaría la monogamia, que es un vínculo afectivo entre dos personas en el que se asume la exclusividad sexual y afectiva; y en el otro, la anarquía relacional, que concibe las relaciones como redes afectivas en las que existen multitud de vínculos de diferente índole afectiva y sexual sin exclusividad o etiquetas (Vasallo, 2020). El poliamor se encontraría en un punto medio en este espectro, e incluye formas muy diversas de establecer vínculos afectivos, con distintos niveles de jerarquización (De las Heras, 2019).

A pesar de la escasez de investigación científica en torno a los estilos de amor en relaciones no normativas, se ha observado que las personas con estilos relacionales no-monógamos también presentan menos niveles de creencias y valores asociados al amor romántico tradicional, desafiando las convenciones sociales asociadas a los estereotipos clásicos de las relaciones de pareja (Klesse, 2006). Esta tendencia a relacionarse según las concepciones tradicionales del amor romántico suele presentarse principalmente en estilos como Storge, Mania y Pragma, de modo que podría esperarse que existiera una mayor prevalencia de esos estilos en personas con relaciones monógamas (Raffagnino \& Puddu, 2018).

Por otro lado, se ha estudiado la relación que presentan los estilos de amor con el bienestar subjetivo (Raffagnino \& Puddu, 2018). Se ha asociado el estilo Eros y Storge, estilos primarios que se relacionan con la atracción física y altos niveles de intimidad y compromiso respectivamente, con mayores niveles de bienestar subjetivo, y Mania, un estilo asociado a la posesividad y los celos, con niveles más reducidos (Kansky, 2018). En cuanto a la relación entre la orientación afectiva y el bienestar, si bien las relaciones no-monógamas se han relacionado con la apertura y el bienestar psicológico, no existe un consenso en literatura en cuanto a las diferencias entre personas monógamas y no-monógamas en esta variable (Sizemore \& Olmstead, 2017).

El estudio del amor romántico en relación al bienestar ha sido estudiado en profundidad en las últimas décadas, pero generalmente desde la misma perspectiva en este contexto sociocultural. La actualización de estos estudios y la inclusión de otras realidades que hasta ahora no han sido abordadas en la investigación es esencial para lograr un estándar de rigor científico adecuado y a la vez dar voz a las minorías que han sido tradicionalmente excluidas de los estudios sobre relaciones interpersonales (Rothschild, 2018).

Este vacío en la literatura acerca de la relación del bienestar con los estilos de amor y la diversidad afectiva ha inspirado el objetivo de esta investigación. El presente estudio tiene dos objetivos principales: (1) analizar la relación entre los estilos de amor y el bienestar y (2) analizar las diferencias en función de la orientación afectiva (personas con parejas monógamas y no-monógamas) en estas variables. 


\section{Método}

\section{Participantes}

Participaron 167 personas con pareja ( $82 \%$ mujeres), cisgénero y con edades comprendidas entre los 16 y los 48 años $(M=26.24$; DT=5.78). En el 88\% su relación de pareja era monógama, y el $22 \%$ presentaban vínculos no monógamos.

\section{Variables e instrumentos}

Las variables sociodemográficas se analizaron mediante un cuestionario ad hoc, en el que se preguntó por género, edad y orientación afectiva, entre otras variables.

Los estilos de amor se evaluaron mediante la Escala de actitudes sobre el amor (Love Attitudes Scale, LAS; Hendrick et al., 1998). Esta escala está compuesta por 18 ítems en escala Likert de 5 puntos, siendo 1 "Completamente en desacuerdo" y 5 "Completamente de acuerdo". Está dividida en 6 factores de 3 ítems cada uno, que corresponden a los seis estilos amatorios concebidos por Lee (1977): tres primarios (Eros, Ludus y Storge) y tres secundarios (Agape, Mania y Pragma).

\section{Procedimiento y análisis}

El estudio se realizó siguiendo las directrices de la declaración de Helsinki (World Medical Association, 2013). Se realizó una encuesta a través de Limesurvey mediante la que se recogieron los datos de todas las personas participantes. Se realizaron análisis descriptivos, correlaciones de Pearson, análisis de regresión lineal jerárquica y pruebas $\mathrm{T}$ para analizar las diferencias de medias.

\section{Resultados}

\section{Correlaciones}

Las correlaciones de Pearson muestran que los estilos de amor están relacionados con el bienestar (ver tabla 1). La satisfacción con la vida está asociada positivamente con Eros $(r=.16, p=.03)$ y negativamente con Mania $(r=.16, p<.01)$ y Agape $(r=-.27$, $p<.01)$. Asimismo, los afectos positivos se asocian positivamente con Eros $(r=.26$, $p<.01)$ y negativamente con Ludus $(r=-.21, p=.01)$, Mania $(r=-.24, p<.01)$ y Agape $(r=$ $-.18, p=.04)$. El afecto negativo se asocia negativamente con Eros $(r=-.18, p=.03) \mathrm{y}$ positivamente con Mania $(r=.33, p<.01)$, y el balance afectivo se relaciona positivamente con Eros $(r=.25, p<.01)$ y negativamente con Ludus $(r=-.13, p=.02) \mathrm{y}$ Mania $(r=-.32, p<.01)$.

\begin{tabular}{|c|c|c|c|c|c|}
\hline EROS & LUDUS & STORGE & PRAGMA & MANIA & AGAPE \\
\hline
\end{tabular}




\begin{tabular}{lcccccc}
\hline $\begin{array}{l}\text { Satisfacción } \\
\text { con la vida }\end{array}$ & $.16^{*}$ & -.04 & .04 & .065 & $-.24^{* *}$ & $-.27^{* *}$ \\
$\begin{array}{l}\text { Afecto } \\
\text { positivo }\end{array}$ & $.26^{* *}$ & $-.21^{*}$ & -.13 & -.03 & $-.24^{* *}$ & $-.18^{*}$ \\
$\begin{array}{l}\text { Afecto } \\
\text { negativo }\end{array}$ & $-.18^{*}$ & .15 & .10 & -.04 & $.33^{* *}$ & .09 \\
$\begin{array}{l}\text { Balance } \\
\text { hedónico }\end{array}$ & $.25^{* *}$ & $-.20^{*}$ & -.13 & .01 & $-.32^{* *}$ & -.15 \\
\hline$* p<.05 .{ }^{* *} p<.01 .^{* * *} p<.001$. & & & &
\end{tabular}

\section{Análisis de regresión lineal jerárquica}

La relación entre los estilos de amor y el bienestar se analizó mediante un análisis de regresión múltiple jerárquica para cada dimensión del bienestar subjetivo (ver tabla 2). En el primer paso, se incluyeron las variables demográficas para controlar el impacto del género y la edad. En el segundo paso, se introdujeron los estilos de amor: Eros, Ludos, Storge, Pragma, Mania y Agape.

El análisis de regresión de la satisfacción con la vida fue significativo en el primer paso, con las variables sociodemográficas $\left(R^{2}=.03, p=.03\right)$, y en el segundo paso aumentó la capacidad predictiva al introducirse los estilos de amor $\left(R^{2}=.11, p<.01\right)$. Los predictores significativos fueron Eros, en sentido positivo $(\beta=.19 ; p<.01)$ y Agape, en sentido negativo $(\beta=-.21 ; p<.01)$. En cuanto a los afectos positivos, el modelo solo resultó significativo al introducirse los estilos de amor, que aumentaron la capacidad predictiva hasta un $12 \%\left(R^{2}=.12, p<.01\right)$. El único predictor significativo fue Eros, en sentido positivo $(\beta=.27 ; p<.01)$. Los afectos negativos fueron explicados por los estilos de amor hasta un $11 \%$, siendo el modelo significativo solo cuando se añadió esta variable en el segundo paso $\left(R^{2}=.11, p<.01\right)$. El único predictor significativo fue Mania, en sentido positivo $(\beta=.38 ; p<.001)$.

Tabla 2

Regresión múltiple jerárquica para las variables predictoras del ajuste psicológico.

\begin{tabular}{|c|c|c|c|c|}
\hline- & $R^{2}$ & $F$ & $\beta$ & $\bar{t}$ \\
\hline \multicolumn{5}{|c|}{ Satisfacción con la vida } \\
\hline Paso 1 & .03 & $3.79 *$ & & \\
\hline Género & & & -.05 & -.59 \\
\hline Edad & & & -.11 & -1.43 \\
\hline Paso 2 & .11 & $3.64 * *$ & & \\
\hline Eros & & & .19 & $2.47 * *$ \\
\hline Ludus & & & .04 & .50 \\
\hline Storge & & & .09 & 1.20 \\
\hline Pragma & & & .07 & .96 \\
\hline Mania & & & -.14 & -1.59 \\
\hline Agape & & & -.21 & $-2.43 * *$ \\
\hline \multicolumn{5}{|c|}{ Afectos positivos } \\
\hline Paso 1 & .01 & 1.56 & & \\
\hline Género & & & -.04 & -.49 \\
\hline
\end{tabular}




\begin{tabular}{|c|c|c|c|c|}
\hline Edad & & & -.05 & -.53 \\
\hline Paso 2 & .12 & $3.27 * *$ & & \\
\hline Eros & & & .27 & $3.24 * *$ \\
\hline Ludus & & & -.12 & -1.42 \\
\hline Storge & & & -.08 & -.98 \\
\hline Pragma & & & -.03 & -.38 \\
\hline Mania & & & -.12 & -1.25 \\
\hline Agape & & & -.10 & -1.02 \\
\hline \multicolumn{5}{|c|}{ Afectos negativos } \\
\hline Paso 1 & -.02 & .01 & & \\
\hline Género & & & .03 & .35 \\
\hline Edad & & & -.10 & -1.10 \\
\hline Paso 2 & .11 & $3.21^{* *}$ & & \\
\hline Eros & & & -.16 & -1.92 \\
\hline Ludus & & & .01 & .12 \\
\hline Storge & & & .10 & 1.23 \\
\hline Pragma & & & -.08 & -1.00 \\
\hline Mania & & & .38 & $3.81^{* * *}$ \\
\hline Agape & & & -.07 & -.75 \\
\hline
\end{tabular}

\section{Diferencias de medias}

Se realizaron pruebas T para analizar las diferencias de medias entre personas con relaciones monógamas y no monógamas en función de los estilos de amor y el bienestar.

Las personas monógamas presentaron mayores niveles en las variables Eros $(t=2.77, p<.01)$, Ludus $(t=2.52, p=.02)$ y Pragma $(t=5.00, p<.01)$. No se observaron diferencias en los niveles de satisfacción con la vida, afectos positivos o afectos negativos.

\section{Discusión}

El presente estudio contaba con dos objetivos: (1) analizar la relación entre los estilos de amor y el bienestar y (2) analizar las diferencias en función de la orientación afectiva (personas con parejas monógamas y no-monógamas) en estas variables.

En cuanto al primer objetivo, se ha observado que los estilos de amor están relacionados con el bienestar. El estilo de amor Eros se asocia positivamente con la satisfacción con la vida, los afectos positivos y el balance hedónico, y negativamente con los afectos negativos, explicando parte de la varianza de estas variables (Kansky, 2018). Estas relaciones van en la línea de la literatura, que indica que este arquetipo amatorio suele asociarse con sentimientos positivos en la pareja o parejas, un estilo de apego seguro, estrategias positivas en regulación de conflictos y satisfacción en la relación, de forma que las personas que presentan más niveles de Eros en sus relaciones afectivo-sexuales también tienden a presentar mayores niveles de bienestar subjetivo 
(Raffagnino \& Puddu, 2018). Ocurre al contrario con Ludus, Mania y Agape, que se relacionan negativamente con la satisfacción con la vida, los afectos positivos y el balance hedónico, y negativamente con los afectos negativos, lo que también va en la línea de la literatura. Ludus y Mania se han asociado a conductas desadaptativas en las relaciones de pareja, formas agresivas de comunicación y tendencia a alimentar emociones negativas como los celos, además de presentar dinámicas de dominancia y posesividad que se asocian con la insatisfacción con las relaciones y bajos niveles de bienestar (Kansky, 2018; Raffagnino \& Puddu, 2018).

Estos resultados sugieren que la atracción y la pasión (propias del estilo Eros) en las relaciones afectivo-sexuales pueden predecir el bienestar psicológico, pero es necesario analizar más variables que puedan jugar un papel en esta relación y que contribuyan a mostrar una imagen más completa de este modelo. Por ejemplo, la duración de la relación podría ser un factor determinante a la hora de explicar esta asociación: esta intensidad emocional suele encontrarse en los primeros estadios de enamoramiento, y tiende a decrecer con el tiempo, por lo que sería interesante explorar la evolución del bienestar subjetivo en relaciones a largo plazo. Por otro lado, la relación negativa entre Mania y bienestar puede explicarse por la relación entre este estilo amatorio y las conductas desadaptativas en las relaciones de pareja; sin embargo, es importante interpretar los resultados con cautela, especialmente a la hora de explicar la relación negativa entre el bienestar y otros estilos como Agape y Ludus. Los factores clave a la hora de explicar el bajo bienestar en relaciones en las que predomina el estilo de Mania no aparecen en otros estilos, pues cada uno presenta características únicas que determinan su relación con el bienestar. Por ejemplo, en el estilo Agape, que se caracteriza por la tendencia a presentar un amor desinteresado y altruista, quizás podría relacionarse negativamente con el bienestar en aquellas relaciones en las que se descuiden las propias necesidades emocionales y este estilo tienda al establecimiento de vínculos poco saludables. En cuanto a Ludus, este estilo se ha asociado principalmente al establecimiento de vínculos fugaces, basados en la atracción estética, y con formas desadaptativas de comunicación que explicarían los bajos niveles de bienestar en personas que presenten estos vínculos.

En cuanto al segundo objetivo, se ha observado que las personas monógamas presentan mayores niveles en los estilos Eros, Ludus y Pragma que las personas no-monógamas. Estos resultados van parcialmente en línea con la literatura, ya que se ha relacionado la tendencia a establecer relaciones monógamas con las concepciones tradicionales del amor romántico, como sería en el caso de Ludus y Pragma, que suelen construirse en torno al ideal de relación de pareja que predomina en los medios tradicionales (Klesse, 2006). Sin embargo, los mayores niveles de Eros no coinciden con esta teoría, lo que sugiere que tal vez la orientación afectiva en sí misma no es suficiente para definir las dinámicas que se establecen en las relaciones de pareja, y que hay más factores implicados que explicarían estas diferencias. Respecto al bienestar, se ha observado que no existen diferencias entre los niveles en esta variable entre personas monógamas y no-monógamas (Sizemore \& Olmstead, 2017). Estos resultados indican que los niveles de bienestar son independientes de el tipo de vínculo que se establezca en las relaciones afectivo-sexuales, y que quizás son otras variables las que determinen el bienestar a lo largo de todo el espectro de la orientación afectiva.

A modo de conclusión, estos resultados muestran que la orientación afectiva puede ser un factor importante a la hora de estudiar el bienestar en las relaciones afectivo-sexuales, pero son otras variables las que contribuyen a interpretar si estos vínculos son saludables. La comunicación, la confianza, la intimidad y la pasión son 
elementos que determinan el bienestar en las relaciones de pareja independientemente del estilo relacional: pueden estar presentes tanto en relaciones monógamas como en el amplio abanico de la no-monogamia, y van más allá de la identificación de los vínculos con una etiqueta (relación abierta, poliamor...) o de los estilos amatorios predominantes en la relación (Rothschild, 2018).

La aparición de los estilos relacionales no normativos en los medios tradicionales ha permitido su visibilidad, y es gracias a que se ha dado voz a estas realidades que se contemplan como una opción más allá de la monogamia (Vasallo, 2020). Es importante que haya una mayor atención a la educación en diversidad afectiva para eliminar los mitos y estereotipos que giran en torno a las relaciones afectivo-sexuales: desde las concepciones hegemónicas del amor romántico que se han vinculado tradicionalmente a la monogamia hasta las creencias erróneas que se tienen acerca de los vínculos existentes en la no-monogamia. Se trata de formas distintas de relacionarse, pero todas estas opciones pueden dar lugar a relaciones saludables $y$ funcionales independientemente de la naturaleza de estos vínculos.

Este estudio presenta ciertas limitaciones. Estos resultados han de ser interpretados con cautela debido al reducido número de participantes en relaciones no-monógamas, por lo que se recomienda replicar este estudio con una muestra más equilibrada y representativa de la población a estudiar. Además, se trata de un estudio transversal, por lo que es recomendable la realización de diferentes evaluaciones a lo largo del tiempo para la aplicación de un diseño longitudinal que pueda estudiar cómo evolucionan estas variables con el tiempo. Por otro lado, se han utilizado cuestionarios de autoinforme que están sujetos a sesgos de respuesta, por lo que es recomendable la utilización de medidas objetivas que puedan complementar la utilización de estos instrumentos y garantizar el rigor de este estudio. Por último, debido a la antigüedad del cuestionario utilizado para evaluar los estilos de amor, es posible que no represente adecuadamente las formas de relacionarse a nivel afectivo-sexual en la actualidad. En futuros estudios se recomienda la utilización de medidas actualizadas y que vayan en línea con la percepción de las relaciones de pareja en la sociedad actual.

A pesar de estas limitaciones, el presente estudio supone una contribución a la literatura en cuanto a que ayuda a visibilizar realidades más allá de las que se presentan como dominantes en este contexto cultural. Todos los estilos relacionales, dentro o fuera de la monogamia, son opciones válidas a la hora de establecer vínculos afectivo-sexuales, y son otros factores los que determinan el bienestar en las relaciones, de la naturaleza que sean. Además, pone de manifiesto el vacío en literatura en materia de diversidad, y la importancia que existe a día de hoy de dar voz a realidades poco visibles que no encajan en las concepciones cisheteronormativas de los vínculos humanos. Es necesario incluir poblaciones diversas en la investigación científica en sexualidad, no solo en cuanto a su orientación afectiva, sino a nivel de identidad de género, orientación sexual, nivel socioeconómico, diversidad funcional, étnica o en personas mayores. La inclusión de estos colectivos contribuiría a derribar mitos prevalentes en la sociedad, y a tener en cuenta la diversidad de experiencias y realidades a la hora de diseñar programas de intervención en materia de sexualidad. La atención a las necesidades específicas de estas poblaciones contribuiría a promover su bienestar psicológico y a disminuir los problemas de salud mental tan prevalentes en colectivos minoritarios. 


\section{Referencias}

De las Heras, R. (2019). Thinking Relationship Anarchy from a Queer Feminist Approach. Sociological Research Online, 24(4), 644-660.

https://doi.org/10.1177/1360780418811965

Gressgård, R. (2013). Asexuality: From pathology to identity and beyond. Psychology and Sexuality, 4(2), 179-192. https://doi.org/10.1080/19419899.2013.774166

Hendrick, S. S., Dicke, A., y Hendrick, C. (1998). The Relationship Assessment Scale. Journal of Social and Personal Relationships, 15(1), 137-142.

https://doi.org/10.1177/0265407598151009

Jonason, P. K., y Kavanagh, P. (2010). The dark side of love: Love styles and the Dark Triad. Personality and Individual Differences, 49(6), 606-610. https://doi.org/10.1016/j.paid.2010.05.030

Kansky, J. (2018). What's Love Got to Do With it? Romantic Relationships and Well-Being. In Handbook of well-being (pp. 1-24). DEF Publishers.

Klesse, C. (2006). Polyamory and its "others": Contesting the terms of non-monogamy. Sexualities, 9(5), 565-583. https://doi.org/10.1177/1363460706069986

Lee, J. A. (1977). A Typology of Styles of Loving. Personality and Social Psychology Bulletin, 3(2), 173-182. https://doi.org/10.1177/014616727700300204

Proctor, C. L., Linley, P. A., y Maltby, J. (2009). Youth life satisfaction: A review of the literature. Journal of Happiness Studies, 10(5), 583-630.

https://doi.org/10.1007/s10902-008-9110-9

Raffagnino, R., y Puddu, L. (2018). Love Styles in Couple Relationships: A Literature Review. Open Journal of Social Sciences, 06(12), 307-330. https://doi.org/10.4236/jss.2018.612027

Ronen, T., Hamama, L., Rosenbaum, M., y Mishely-Yarlap, A. (2016). Subjective well-being in adolescence: The role of self-control, social support, age, gender, and familial crisis. Journal of Happiness Studies, 17(1), 81-104. https://doi.org/10.1007/s10902-014-9585-5

Rothschild, L. (2018). Compulsory Monogamy and Polyamorous Existence. Graduate Journal of Social Science, 14, 28-56. Recuperado de www.amazon.com

Sánchez-Álvarez, N., Extremera, N., y Fernández-Berrocal, P. (2015). Maintaining life satisfaction in adolescence: Affective mediators of the influence of perceived emotional intelligence on overall life satisfaction judgments in a two-year longitudinal study. Frontiers in Psychology, 6, 1892.

https://doi.org/10.3389/fpsyg.2015.01892

Schoeps, K., Montoya-Castilla, I., y Raufelder, D. (2019). Does Stress Mediate the Association Between Emotional Intelligence and Life Satisfaction During Adolescence? Journal of School Health, 89(5), 354-364. https://doi.org/10.1111/josh.12746 
Sheff, E., \& Tesene, M. M. (2015). Consensual Non-Monogamies in Industrialized Nations. https://doi.org/10.1007/978-3-319-17341-2_13

Sizemore, K. M., y Olmstead, S. B. (2017). A systematic review of research on attitudes towards and willingness to engage in consensual non-monogamy among emerging adults: methodological issues considered. Psychology \& Sexuality, 8, 4-23. https://doi.org/10.1080/19419899.2017.1319407

Thorne, S. R., Hegarty, P., y Hepper, E. G. (2019). Equality in theory: From a heteronormative to an inclusive psychology of romantic love. Theory and Psychology, 29(2), 240-257. https://doi.org/10.1177/0959354319826725

Vasallo, B. (2020). Pensamiento monógamo, terror poliamoroso (La Oveja R). Recuperado de https://www.amazon.es/Pensamiento-monógamo-poliamoroso-Brigitte-Vasallo/dp/ 8416227241

Walsh, A. (2017). Love: The Biology Behind the Heart (Routledge). Recuperado de https://books.google.es/books?hl=es\&lr=\&id=Ky0rDwAAQBAJ\&oi=fnd\&pg=PA $175 \& d q=$ love + styles\&ots $=$ rW1qNcdM2Z\&sig=ZAkiHdgymxRCNqE_KeFrL-8W $\mathrm{g} \_\mathrm{g} \# \mathrm{v}=$ onepage $\& \mathrm{q}=$ love styles $\& \mathrm{f}=$ false

World Medical Association. (2013). World Medical Association Declaration of Helsinki: ethical principles for medical research involving human subjects. Journal of American Medical Association, 310, 2191-2194. 